\title{
75 Jahre Röntgeninstitut der Charité
}

Festrede von Herrn Prof. Walter Reisinger, emeritierter Itd. Oberarzt der Radiologie am Campus Mitte der Charité anlässlich des 75-jährigen Bestehens des Röntgeninstituts der Charité auf dem Wissenschaftssymposium am 11. Juli 2014 in Berlin mit einem Grußwort von Roland Felix, Meinhard Lüning und Karl-Jürgen Wolf.

Die Geschichte der Radiologie in Berlin ist mit etwa 118 Jahren deutlich älter als die des Röntgeninstituts der Charité mit seinen 75 Jahren. Lange vor seiner Gründung waren röntgenologisch tätige Ärzte in Berliner Krankenhäusern und Privatinstituten erfolgreich tätig. Hier nur einige wenige Bespiele: Der Physiker und Mediziner Gustav Bucky, Erfinder der nach ihm benannten Streustrahlenblende, kam 1908 an das Rudolf-Virchow-Krankenhaus.

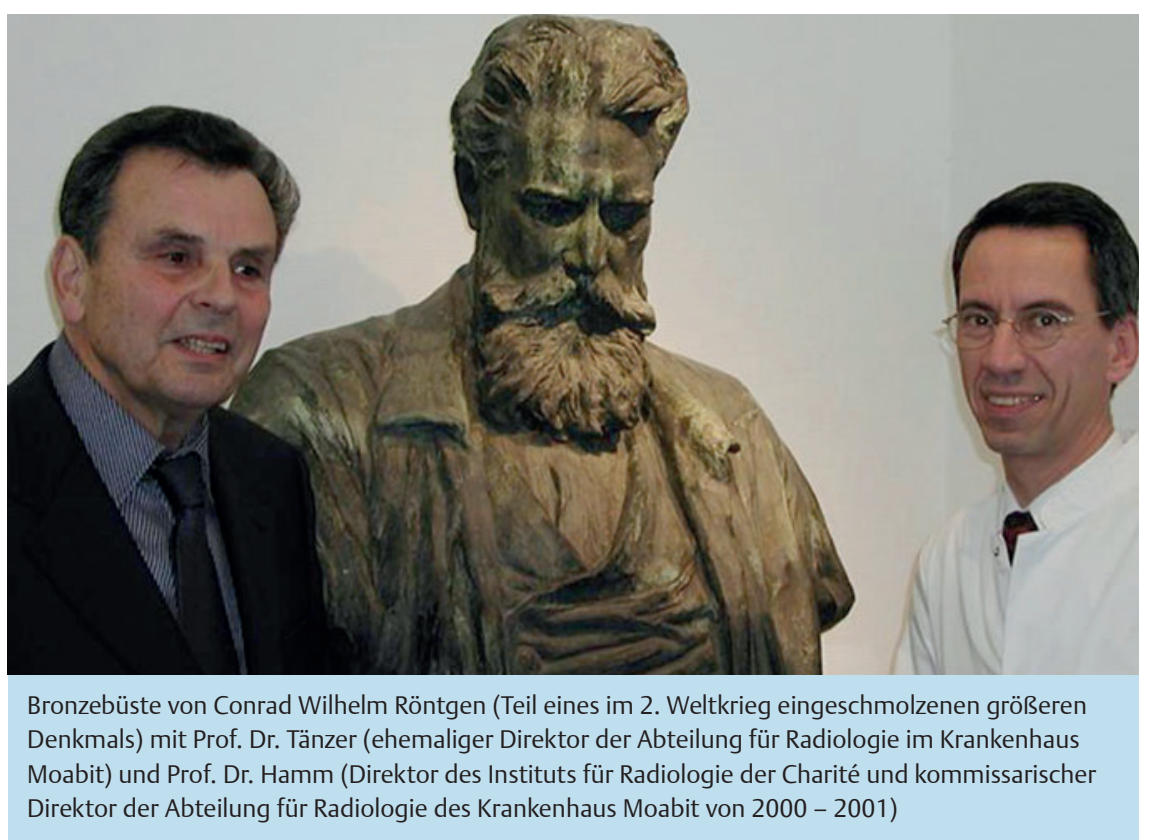

Dort hatte der Chirurg Max Levy-Dorn im Jahre 1903 ein „Zentralinstitut für Untersuchungen und Behandlungen mit Röntgenstrahlen“ eingerichtet. Levy-Dorn war im Jahre 1898 auch Mitbegründer der „Röntgenvereinigung zu Berlin“, der 1. und heute damit ältesten deutschsprachigen Röntgengesellschaft. Im Krankenhaus Moabit leitete seit 1923 Karl Frik das Werner-von-Siemens-Institut für Röntgenforschung. Der Deutsch-Libanese Henri Cha- oul - Stichwort „Chaoulsche Nahbestrahlung “ - war in der chirurgischen Klinik der Charité röntgenologisch tätig, dann im Krankenhaus Moabit. Chaouls Einsatz während des 2. Weltkriegs verdanken wir die Rettung unserer heute im Campus Charité Mitte stehenden Bronzebüste Conrad Wilhelm Röntgens.

\section{Von 1939 bis heute \\ $\nabla$}

Eine umfassende Darstellung der Entwicklung des Röntgeninstituts der Charité ist heute natürlich nicht möglich. Nur wesentliche Teile sollen deshalb episodenhaft geschildert werden.

Die Gründung des Instituts am 3. Juli 1939 fällt in die dunkelste Zeit der deutschen Geschichte, in die des Nationalsozialismus. Es ist einleuchtend, dass man sich mit einer Würdigung dieses Jubiläums zunächst schwer tut. Unser Institut veranstaltet daher sinnvollerweise ein Wissenschaftssymposium. Es ist sicher die klügste Entscheidung, so mit einem eigentlich ungeliebten Gründungsdatum umzugehen.

Analysiert man die 75 Jahre des Bestehens des Instituts, sind mehrere Zeitzonen abzugrenzen. Eine 1. Phase von der Grün- 
dung 1939 bis zum Kriegsende 1945, mit allen Zwängen und zerstörerischen Folgen der nationalsozialistischen Diktatur. Dann eine Kriegstrümmer beseitigende und zunächst antifaschistisch-demokratisch geprägte Nachkriegsphase im sowjetisch besetzten Ostberlin. Der Gründung der DDR im Jahre 1949 folgt die 40-jährige DDR-Zeit mit den Zeichen einer erneuten Diktatur einerseits, aber mit für die Charité und das Röntgeninstitut bedeutenden baulichen und technischen Veränderungen andererseits. Dann die Zeit nach der politischen Wende 1989. Die Charité und ihr Röntgeninstitut kämpfen erfolgreich um das reale Überleben als wissenschaftliche Institution. Das Wiedererstarken des Instituts kulminiert in der Fusion der radiologischen Einrichtungen der Campi Rudolf-Virchow-Klinikum und Klinikum Benjamin Franklin zur Charité. Kurz gesagt: Mehr als 2/3 seiner 75-jährigen Geschichte musste das Institut in Gesellschaftssystemen bestehen, die nicht freiheitlich-demokratischen Grundsätzen entsprachen. Daran sollte man stets denken, wenn nun einige wesentliche Aspekte in der interessanten und lehrreichen Geschichte unseres Instituts genannt werden.

\section{Die Anfänge: Ferdinand Sauer- bruch und Karl Frik \\ $\nabla$}

Am Anfang war das Wort eines Chirurgen, er begründete unsere Geschichte! Der im Jahre 1927 an die Charité berufene Ferdinand Sauerbruch plante im Jahre 1938 den Neubau eines Operationstraktes für seine chirurgische Klinik. Seine radiologisch tätigen Mitarbeiter Henry Chaoul, Walter Cowl und Rudolf Grashey bezog er in die Planungen hinsichtlich der Neugestaltung der Röntgenabteilung ein. Sauerbruch wollte, und das ist die eigentliche Geburtsstunde einer eigenständigen Radiologie an der Charité, an Stelle der bisher bestehenden Röntgenabteilung ein eigenständiges Röntgeninstitut begründen, verbunden mit dem 1 . Lehrstuhl für Radiologie in Berlin. Die Gründung des Instituts mit dem Namen „Universitätsinstitut für Röntgenologie und Radiologie und Strahlentherapeutische Klinik“" erfolgte am 3. Juli 1939. Und das ist für uns Radiologen die eigentliche Großtat der sonst nicht unumstrittenen Person des Ferdinand Sauerbruch. Vielleicht ist das der Grund für unser stets gutes Verhältnis zu den Chirurgen. Erster Direktor und gleich-

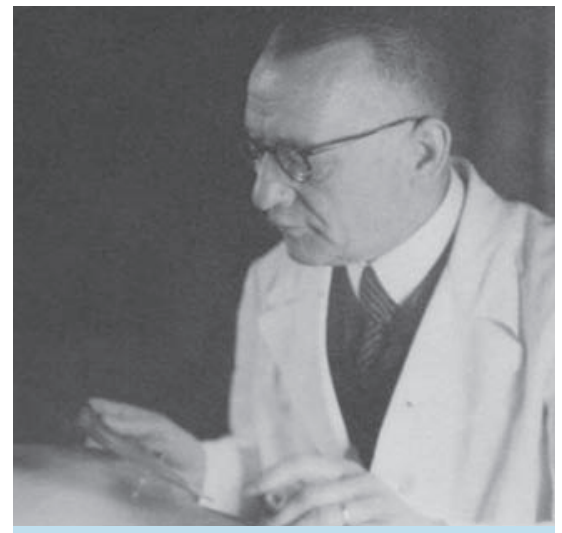

Karl Frick (1878 - 1944)

zeitig Inhaber des neu eingerichteten Lehrstuhls Röntgenologie wurde Karl Frik.

Frik hatte zuvor, wie erwähnt, das Werner-von-Siemens-Institut des Krankenhauses Moabit geleitet. Im neu erbauten Nordost-Flügel der Chirurgischen Klinik bezog das Röntgeninstitut Untersuchungs- und Arbeitsräume im Souterrain und im 1. Obergeschoss, unter dem Sauerbruchschen Operationstrakt.

Bei der Gründung des Instituts wurde ein sogenanntes „Hausbuch“ angelegt. Es gibt Auskunft über die Institutsangehörigen. Dort finden sich beispielsweise bekannte Namen wie Heinz Oeser und Lothar Diethelm. Die von Frik handschriftlich verfasste einleitende Widmung ist frei vom damaligen Zeitgeist und wert, heute noch gelesen zu werden. Ich gebe zu, dass mich der Text beeindruckt hat, er soll deshalb den Abschluss meines Beitrags bilden.

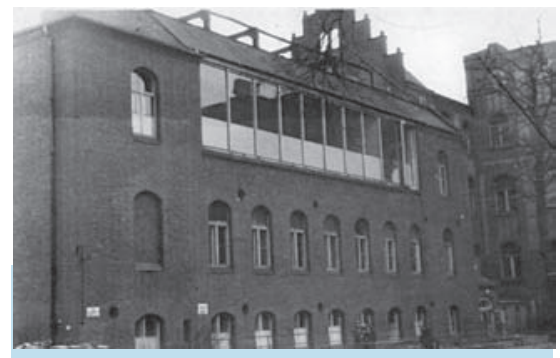

Röntgeninstitut in der Chirurgischen Klinik 1950

\section{Kriegsende und Nachkriegszeit} $\nabla$

Obwohl Karl Frik bis 1939 Präsident der Deutschen Röntgengesellschaft war und dann bis zu seinem Tode 1944 Direktor der renommiertesten Röntgeneinrichtung Deutschlands, war er - im Gegensatz $\mathrm{zu}$ einigen seiner damaligen Kollegen nicht Mitglied der NSDAP. Sein Nachfolger wurde in den letzten Kriegsmonaten Henri Chaoul. Er kam aus dem Krankenhaus Moabit an seine alte Wirkungsstätte zurück, verließ allerdings kurz vor Kriegsende Berlin. Auf Anregung Sauerbruchs wurde Ende 1945 der bereits 70-jährige Rudolf Grashey Lehrstuhlinhaber. Grashey musste aber krankheitshalber 1949 die Institutsleitung aufgeben. Von 1949 bis 1951 wurde das Institut kommissarisch geleitet. Hauptaufgabe in diesen schweren Nachkriegszeiten war die Wiederherstellung der diagnostischen und therapeutischen Kapazitäten. 1951 wurde Fritz Gietzelt, ein Vertreter der Konzentration der Radiologie, aus Leipzig auf den Lehrstuhl nach Berlin berufen. Sein Hauptinteresse galt dem Aufbau der Geschwulstklinik auf der Ruine der ehemaligen 2. Charité-Frauenklinik an der Invalidenstraße. Dort konzentrierte er die Strahlentherapie sowie die dazu nötige Röntgendiagnostik.

Im Röntgeninstitut verblieb zunächst nur die Röntgendiagnostik für die Chirurgie. Aber im Jahre 1959 wurden dem Institut dann auch die Röntgendiagnostik der Geschwulstklinik sowie die bisher separaten Röntgenabteilungen aller operativen Kliniken, der Hautklinik und der Stomatologie zugeordnet. Direktor dieses nun deutlich größeren, nur noch diagnostisch tätigen Instituts wurde im Jahre 1959 ein Oberarzt Gietzelts, Günther Ließ. Eigenständig blieben die Röntgenabteilungen der I. und II. Medizinischen Klinik und Poliklinik sowie die der Kinder- und der Nervenklinik.

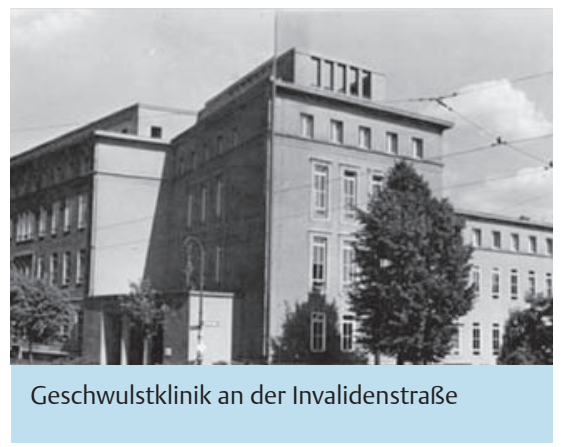

\section{Die Zeit in der DDR}

$\nabla$

Während der DDR-Zeit bekamen Ostberlin und auch die Charité - als Aushängeschild gegenüber dem kapitalistischen Westberlin gedacht - stets eine Spitzenstellung eingeräumt. So entstand der Plan, einen Neubau auf dem Gelände der alten Charité zu errichten. Zur Bauplanung, an 
der alle Kliniken und Institute beteiligt wurden, auch das Röntgeninstitut, seit 1987 unter seinem Direktor Meinhard Lüning, entstand ein Direktorat „Neubau und Rekonstruktion“.

Im Ergebnis entstand das neue Domizil des Röntgeninstituts im Hochhaus und dem über die Brücke erreichbaren Funktionstrakt. Einzug war im Jahre 1981.

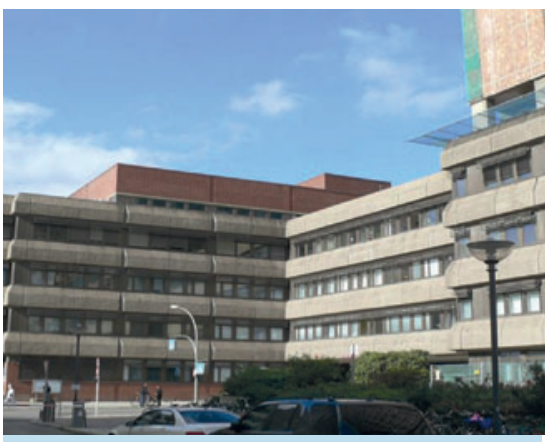

Röntgeninstitut nach 1981

Im Jahre 1978 wurde die bisher separate Röntgenabteilung der Medizinischen Klinik in das nunmehrige „Zentralinstitut“ einbezogen. Eigenständig bis zum Jahre 1989 bzw. 1992 blieben lediglich die Röntgenabteilungen in der Neurologie und der Kinderklinik. Als Sonderfall bleibt zu berichten, dass es auch der Zentralisation entgegengesetzte Bemühungen gab. Der durch seine bahnbrechenden Ergebnisse in der kardio-vaskulären Diagnostik und Therapie weltbekannte Werner Porstmann, ein Oberarzt von Ließ, erreichte Anfang der sechziger Jahre, aus seiner bisherigen Abteilung ein eigenständiges „Institut für Kardiovaskuläre Diagnostik“ zu machen.

Ein Universitätsinstitut muss forschen, das ist neben der Lehre und der Patientenversorgung seine Hauptaufgabe. Wie wurde zu DDR-Zeiten geforscht? Zu jener Zeit - und wie ich meine auch heute noch - war die Radiologie eines der Fachgebiete mit den bedeutendsten Innovationen. Aber Maßstab aller Dinge sind hier nicht nur die intellektuellen Fähigkeiten und der Forscherfleiß der Wissenschaftler, sondern auch die technischen Neuerungen, also moderne bildgebenden Systeme und Computer. Die Charité und das Röntgeninstitut erhielten auch vor der Wende einzelne moderne Geräte - aber mit einer so deutlichen zeitlichen Verzögerung, dass eine Grundlagenforschung praktisch nicht möglich war. Es wurde aber klinische Forschung betrieben und gemeinsam mit westlichen Wissenschaftlern publiziert. Den ersten im Jahre 1979 in der DDR installierten Computertomografen - natürlich westlicher Provenienz - erhielt die Charité. Er stand aber nicht im Röntgeninstitut, sondern in der Nervenklinik. Die Radiologie durfte partizipieren. Doch schon das 2. CT-Gerät kam dann in das Röntgeninstitut. Ihren 1. Kernspintomografen erhielt das Röntgeninstitut im Jahre 1987, er blieb dann bis zur Wende das einzige MRT-Gerät der DDR.

\section{Nach der Wende 1989}

$\nabla$

Zur Geschichte unseres Instituts gehört zum Glück auch die Zeit nach der politischen Wende 1989. Der Wille zu einem Neuanfang nach der unblutig erfolgten Auflösung der DDR war groß, aber die damit verbundenen Veränderungen waren gravierend. Manche ärztlichen Mitarbeiter verließen das Institut, aus den unterschiedlichsten Gründen. Aber viele blieben und nahmen mit den neu Hinzugekommenen das Wagnis auf sich, die Charité und das Röntgeninstitut zu erneuern. Das war nicht einfach. Einige damals führende Gesundheitspolitiker wollten die Charité, wie so viele ostdeutsche Einrichtungen auch, ,abwickeln“. Wir sollten verschwinden, zumindest in die Bedeutungslosigkeit. Es waren dann Mediziner aus ganz Deutschland, Europa und Übersee, welche ihr völliges Unverständnis äußerten zu der Absicht, eine trotz aller Widrigkeiten immer noch weltbekannte Institution aus der deutschen Medizingeschichte streichen zu wollen. Ihren Einfluss geltend machten auch die an die Charité Neuberufenen, die aus renommierten westberliner, westdeutschen und europäischen Kliniken gekommenen Hochschullehrer. $\mathrm{Zu}$ den Ersten zählten die Herren Baumann, Einhäupl und auch unser jetziger Institutsdirektor Bernd Hamm. Und für die Erhaltung der Charité stand auch der damalige Wissenschaftssenator Manfred Erhardt ein. Gemeinsam sicherten wir den Weiterbestand der damals schon beinahe 300 Jahre bestehenden Charité und damit auch den unseres Röntgeninstituts, welches heute in den 3 Campi (Campus Benjamin Franklin, Campus Mitte und Campus Virchow-Klinikum) alle radiologischen Einrichtungen der Freien Universität Berlin und der Humboldt-Universität zu Berlin umfasst.

\section{Danksagung und Blick in die Zu- kunft \\ $\nabla$}

Wenn wir heute auf diesem Symposium an die Gründung unseres Instituts denken mit zahlreichen Vorträgen, die das hohe wissenschaftliche Niveau dieser Einrichtung demonstrieren, sollten wir nicht die vielen MTRA, die Krankenschwestern, die

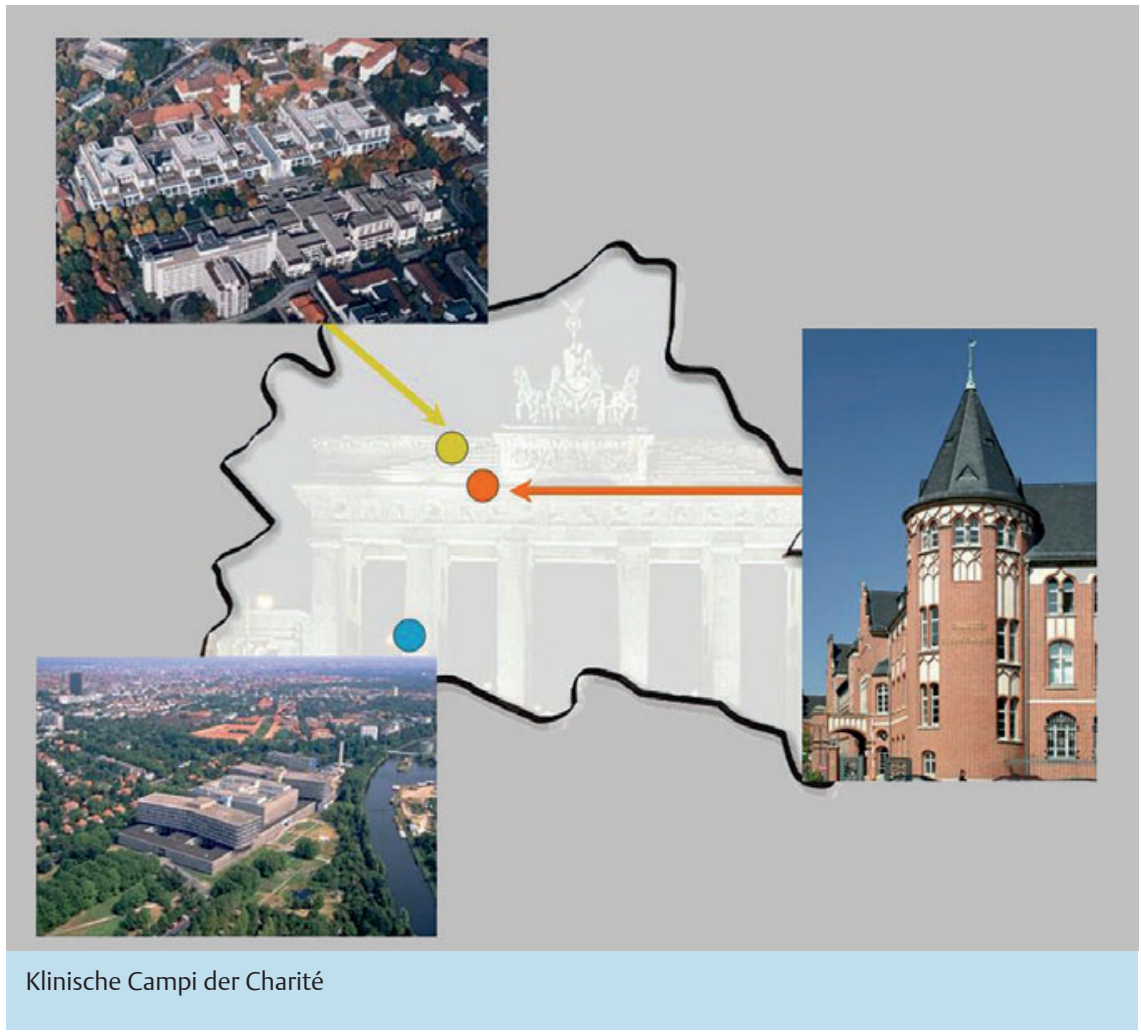


Sekretärinnen, die Anmeldekräfte, im Archiv Tätige und die Reinigungskräfte vergessen. Auch der vielen ebenfalls im Dunkel der Geschichte verbleibenden Ärzte, Physiker und Informatiker sei gedacht, die durch alle Zeiten und unter unterschiedlichen politischen Regimes dennoch mit Einsatzbereitschaft, Freude am Beruf und Hinwendung zu unseren Patienten dem Institut zu seiner Erfolgsgeschichte verholfen haben. Ich wünsche dem Institut weiterhin den Erfolg, der auf sehr guten Leistungen beruht verbunden mit der nötigen Fortune. Und damit verbinde ich auch meinen herzlichsten Wunsch nach einem weiteren Gedeihen unseres national und international hochgeachteten, nun in der ganzen wiedervereinigten Stadt präsenten Instituts.

Lesen Sie nun zum Schluss den Text der Widmung Friks im Gründungsbuch unseres Instituts.

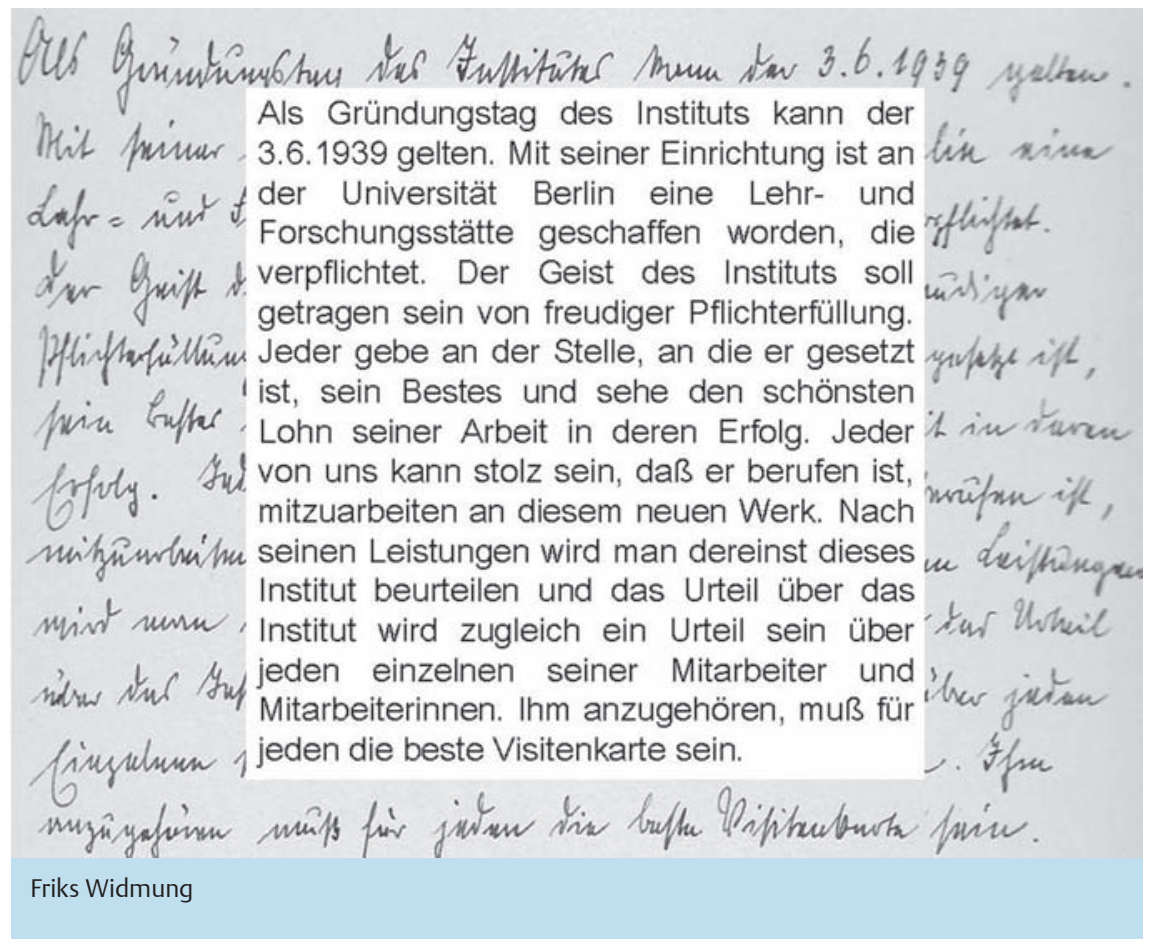

\section{Grußwort von Roland Felix*, Meinhard Lüning* und Karl-Jürgen Wolf* $\nabla$}

( ${ }^{*}$ Emeriti der Klinik für Strahlenheilkunde - Campus Virchow-Klinikum, Institut für Radiologie - Campus Mitte und Klinik für Radiologie und Nuklearmedizin Campus Benjamin Franklin)

„Wir gratulieren allen Wissenschaftler/Innen und Mitarbeiter/Innen der Klinik für Radiologie der Charité zum 75-jährigen Bestehen und wünschen unserer traditionsreichen Institution weiterhin eine besondere Hingabe in Diagnostik und Therapie unserer Patienten, wegweisende Erfolge in der Forschung und eine von den Studenten geschätzte exzellente Lehre." 